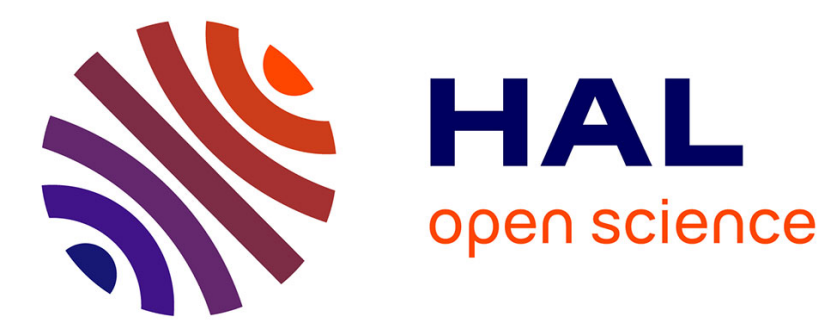

\title{
Outcome of axillary staging in early breast cancer: a meta-analysis
}

Malcolm R. Kell, John P. Burke, Mitchel Barry, Monica Morrow

\section{To cite this version:}

Malcolm R. Kell, John P. Burke, Mitchel Barry, Monica Morrow. Outcome of axillary staging in early breast cancer: a meta-analysis. Breast Cancer Research and Treatment, 2010, 120 (2), pp.441-447. 10.1007/s10549-009-0705-6 . hal-00535431

\section{HAL Id: hal-00535431 \\ https://hal.science/hal-00535431}

Submitted on 11 Nov 2010

HAL is a multi-disciplinary open access archive for the deposit and dissemination of scientific research documents, whether they are published or not. The documents may come from teaching and research institutions in France or abroad, or from public or private research centers.
L'archive ouverte pluridisciplinaire HAL, est destinée au dépôt et à la diffusion de documents scientifiques de niveau recherche, publiés ou non, émanant des établissements d'enseignement et de recherche français ou étrangers, des laboratoires publics ou privés. 


\title{
Outcome of axillary staging in early breast cancer: a meta-analysis
}

\author{
Malcolm R. Kell • John P. Burke • Mitchel Barry • \\ Monica Morrow
}

Received: 15 December 2009/Accepted: 17 December 2009/Published online: 9 January 2010

(C) Springer Science+Business Media, LLC. 2010

\begin{abstract}
Axillary lymph node dissection (ALND) is associated with significant morbidity, whilst sentinel node biopsy (SNB) has the potential to minimize complications in the management of breast cancer. The aim of this study was to systematically appraise the outcome of SNB when compared to ALND. A comprehensive search for published trials examining outcomes after SNB for breast cancer was performed using medline and cross-referencing available data. Each study was reviewed and data extracted. Primary outcomes were nodal positivity and surgery-related morbidity. A total of 9,608 patients were identified from trials comparing ALND and SNB. The overall rate of axillary lymph node positivity for those with no clinically palpable nodes was $28.8 \%$ for ALND and $27.6 \%$ for SNB $(\mathrm{OR}=1.00,95 \% \mathrm{CI}=0.86-1.17, P=0.956)$, though there was a trend for superior detection of metastatic disease with SNB when this was compared with ALND alone $(\mathrm{OR}=1.22,95 \% \mathrm{CI}=0.95-1.57, P=0.122)$. Patients who undergo SNB are significantly less likely to suffer post-operative morbidity relative to ALND: risk of infection $(\mathrm{OR}=0.58,95 \% \mathrm{CI}=0.42-0.80, P=0.0011)$, seroma $(\mathrm{OR}=0.40,95 \% \mathrm{CI}=0.31-0.51, P=0.0071)$, arm swelling $(\mathrm{OR}=0.30,95 \% \mathrm{CI}=0.14-0.66, P=0.0028)$ and numbness $(\mathrm{OR}=0.25,95 \% \mathrm{CI}=0.1-0.59, P=0.0018)$.
\end{abstract}

Presented in part at American Society of Clinical Oncology, Chicago, IL, USA, June 2008.

M. R. Kell $(\bowtie) \cdot$ J. P. Burke · M. Barry

Eccles Breast Screening Unit, Mater Misericordiae University

Hospital, Eccles Street, Dublin 7, Ireland

e-mail: malcolm.kell@breastcheck.ie; malcolm.kell@ucd.ie

M. Morrow

Department of Surgical Oncology, Memorial Sloan Kettering

Cancer Centre, New York, NY, USA
SNB is at least equivalent to ALND in detecting metastatic disease in the axilla. SNB is the optimum approach in terms of morbidity for the assessment of axillary metastasis in clinically node negative breast cancer.

Keywords Sentinel - Axillary - Lymphadenectomy · Outcome $\cdot$ Morbidity

$\begin{array}{ll}\text { Abbreviations } \\ \text { ALND } & \text { Axillary lymph node dissection } \\ \text { SNB } & \text { Sentinel node biopsy } \\ \text { OR } & \text { Odds ratio } \\ \text { CI } & \text { Confidence interval } \\ \text { BCRL } & \text { Breast cancer-related lymphoedema } \\ \text { QUOROM } & \text { Quality of Reporting of Meta-Analyses } \\ \text { df } & \text { Degrees of freedom }\end{array}$

\section{Introduction}

The surgical management of breast cancer has traditionally been comprised of resection of the primary tumour and axillary lymph node dissection (ALND). There are significant short- and long-term morbidities associated with ALND, including seroma formation, impaired shoulder movement, neuropathy and arm lymphedema. As a consequence of population-based screening and the increased use of surveillance mammography, breast cancer is often diagnosed at a smaller size when axillary lymph node metastases are less likely [1]. The majority of women with early stage breast cancer are node negative at the time of diagnosis [2], and ALND in these women exposes them to the complications of this procedure without benefit. Studies of sentinel node biopsy (SNB) followed by axillary 
dissection have established that SNB accurately predicts the status of the other axillary nodes [3], but SNB has a false negative rate of $5-10 \%$ when compared to SNB followed by axillary dissection [3, 4]. However, randomized studies comparing the rate of detection of positive nodes after SNB and ALND alone show equivalent rates of metastasis: therefore ALND must have a comparable false negative rate. This meta-analysis aimed to compare the staging accuracy of these two procedures and document the morbidity of each.

\section{Materials and methods}

Identification of studies

We searched MEDLINE and EMBASE by entering the following in the searching algorithm: sentinel AND breast AND clinical trial OR randomized controlled trial OR random OR randomized. We also searched the Cochrane Central Register of Controlled Trials for randomized trials that compared ALND and SNB. We set English as a language restriction. The latest search was done on April 1st, 2009. Three authors (JPB, MB and MRK) independently examined the title and abstract of citations and the full texts of potentially eligible trials were obtained; disagreements were resolved by discussion. The reference list of retrieved papers was further screened for additional publications.

\section{Eligibility criteria}

Only prospective, randomized controlled trials which directly compared SNB and ALND were selected to ensure comparable patient groups. Only reports on patients with a primary diagnosis of invasive breast cancer with no previous history of breast cancer were included. The primary endpoints of this meta-analysis were nodal positivity and post-operative morbidity.

\section{Data extraction and outcomes}

We recorded the following information regarding each eligible trial: authors' names, journal, year of publication and study design items (including whether there was a description of the mode of randomization, allocation concealment, number of withdrawals per arm and blinding). We recorded the following information from both arms of each eligible trial: the number of patients randomly assigned to treatment and analyzed per arm, median age, size of tumour and number of outcome events per arm (Table 1). The primary outcomes were post-operative morbidity at 6 months following surgery and the chance of having a positive lymph node after SNB or ALND; these data were calculated from the number of positive nodes from those having an acceptable axillary staging procedure.

\section{Statistical analysis}

We estimated the odds ratio (OR), with its variance and 95\% confidence interval (CI). Heterogeneity between the ORs for the same outcome between different studies was assessed by use of the $\chi^{2}$-based $Q$ statistic [5]. Data were then combined across studies by the use of general variance methods with fixed and random effects models [5]. The fixed effects analysis weighted the natural logarithm of each study's OR by the inverse of its variance. The random effects analysis weighted the natural logarithm of each study's OR by the inverse of its variance plus an estimate of the between-study variance in the presence of betweenstudy heterogeneity. In the absence of between-study heterogeneity, fixed and random effects coincide because the between-study variance is zero. Analyses were conducted using Statsdirect version 2.5.6 (StatsDirect Ltd, Cheshire, UK) and SPSS version 12.0 (SPSS, Inc., Chicago, IL). All statistical tests were two-tailed.

\section{Results}

Eligible studies

We identified seven potentially eligible randomized controlled trials that directly compared SNB and ALND (Table 1). Eighty-nine studies were excluded from the meta-analysis (Fig. 1). Seven more trials are ongoing, and no peer-reviewed report has yet been published [6]. No data on post-operative morbidity has yet been published from the NSABP-B32 trial, so this study was included only in the analysis of nodal positivity.

Seven trials were thus eligible, as shown in Table $1[3,4$, 7-11]. A total of 9,608 patients were randomly assigned to treatment (658 in the SNB only arm, 5,084 in the ALND only arm and 4,406 who had SNB and then went onto have ALND), and data was available for analysis from 8,853 of them (636, 4,389 and 3,828 in the three arms, respectively). In studies which reported the average age of participants, the mean age ranged between 54 and 58 years. There was little variability among studies in the menopausal status, eligible stages of breast cancer and tumour size of participants. All seven trials enroled patients between 1998 and 2005, and six of the seven studies were multicentre trials [4, 7-11].

Five trials $[3,4,7,9,11]$ described the method of power calculation, six trials $[4,7-11]$ described in detail the mode of randomization and five trials $[4,7-9,11]$ described the 
Table 1 Characteristics of eligible randomized trials for this meta-analysis

\begin{tabular}{lccclll}
\hline & $\begin{array}{l}\text { No. patients } \\
\text { analyzed }\end{array}$ & SLND & ALND & $\begin{array}{l}\text { Recruitment stage/ } \\
\text { size }(\mathrm{mm})\end{array}$ & $\begin{array}{l}\text { Enrolment } \\
\text { interval (yr) }\end{array}$ & $\begin{array}{l}\text { Country } \\
\text { (no. of centres) }\end{array}$ \\
\hline Milan trial & 516 & 259 & 257 & $<20$ & $1998-1999$ & Italy (1) \\
Purushotham et al. & 298 & 143 & 155 & $<30$ & $1999-2003$ & UK (4) \\
ALMANAC trial & 954 & 478 & 476 & No palpable LN & $1999-2003$ & UK (13) \\
Z0011 trial & 744 & 371 & 373 & T1 or T2, N0, Mo & $1999-2004$ & USA (7) \\
NSABP-B32 trial & 5,316 & 2,697 & 2,619 & No palpable LN & $1999-2004$ & USA and Canada (80) \\
GIVOM trial & 697 & 345 & 352 & $<30$ & $1999-2004$ & Italy (18) \\
SNAC trial & 1,083 & 544 & 539 & $<30$ and no palpable LN & $2001-2005$ & Australia and New Zealand (31) \\
Total & 9,608 & & & & & \\
\hline
\end{tabular}

$L N$ lymph node

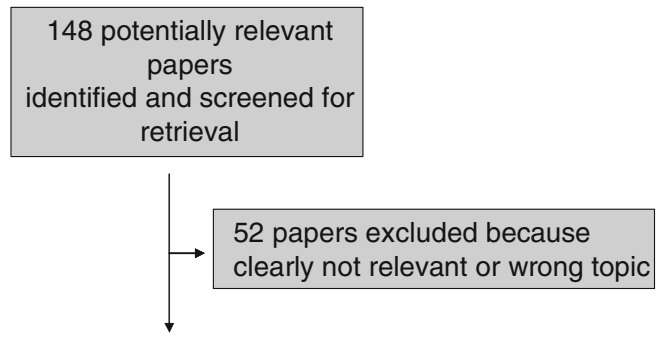

96 retrieved for full text

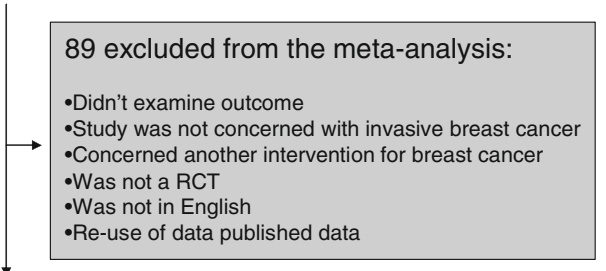

7 studies included in the meta-analysis

Fig. 1 Improving the quality of reports of meta-analyses of randomized controlled trials; the Quality of Reporting of Meta-Analyses (QUOROM) statement flow diagram

mode of allocation concealment in detail. Withdrawals were described in detail in six trials [3, 4, 8-11]. None of the seven studies were blinded. One trial was terminated early due to clear loss of equipoise in relation to morbidity [4] and one ended due to increasing patient withdrawal prerandomization [9], and one was stopped due to slow accrual and a lower than anticipated event rate [7].

\section{Nodal positivity}

In one trial the control group underwent ALND only and the test group underwent SNB followed by routine ALND [7]. In four trials the control group underwent SNB followed by routine ALND and the test group underwent SNB with ALND only if the SNB was positive [3,8-10]. In two trials the control group underwent ALND only and the test group underwent SNB with ALND only if the SNB was positive $[4,11]$. In these studies, there was no significant difference in the rate of axillary lymph node positivity among clinically node negative patients; $28.8 \%$ for ALND and $27.6 \%$ for SNB [ALND $(n=4,464)$ and SNB $(n=4,464) \quad(\mathrm{OR}=1.00, \quad 95 \% \quad \mathrm{CI}=0.86-1.17, \quad P=$ 0.956)] [3, 4, 7, 9-11] (Fig. 2). Examination of trials which compared SNB (without a backup ALND) with ALND only [4, 7] led to a non-significant trend in increased likelihood of having a positive axillary node in patients who underwent SNB $(27.7 \%, n=636)$ when compared to ALND only $(24 \%, n=651)(\mathrm{OR}=1.2295 \% \mathrm{CI}=0.95$ $-1.57, P=0.122)$.

Morbidity

The most concordant complications examined between trials were wound infection and seroma formation at 30 days and numbness and limb swelling at 6 months.

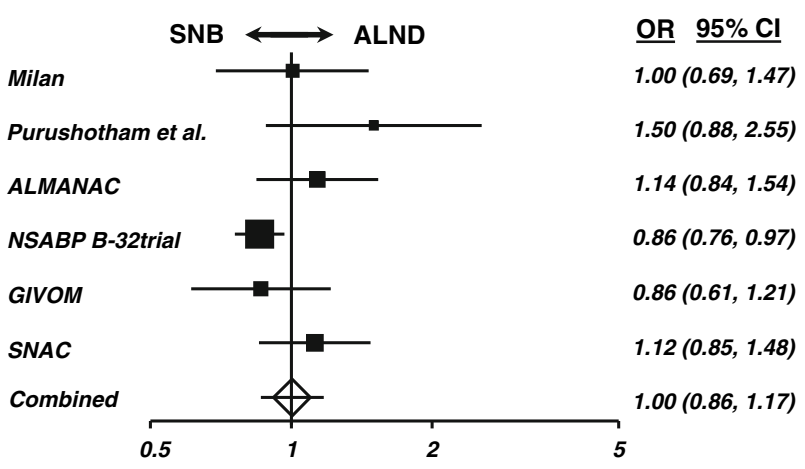

Fig. 2 Meta-analysis for nodal positivity following SNB or ALND. Each study is shown by the point estimate of the odds ratio (square proportional to the weight of each study) and $95 \%$ confidence interval (CI) for the risk ratio (extending lines); the summary odds ratio and 95\% confidence intervals by random effects calculations are also shown by diamonds. SNB (sentinel lymph node dissection), left of unity versus ALND (axillary lymph node dissection), right of unity. $n=8,853, P=0.956$. Test for heterogeneity, $Q=9.45 ;(\mathrm{df}=6)$, $P=0.092 ; I^{2}=47.1 \%$ 
Morbidity at 30 days

In analysis of 2,781 patients from three randomized controlled trials [4, 8, 11], there was a significant reduction in the risk of post-operative wound infection in those patients undergoing $\mathrm{SNB}$ versus ALND $(\mathrm{OR}=0.58, \quad 95 \%$ $\mathrm{CI}=0.42-0.80, P=0.0011)($ Fig. 3a).

A significant reduction in the risk of post-operative seroma was also seen in those patients undergoing a SNB versus $\quad$ ALND $\quad(\mathrm{OR}=0.40, \quad 95 \% \quad \mathrm{CI}=0.31-0.51$, $P=0.0071$ ) (Fig. 3b). This endpoint was reported in 2,125 patients from three randomized controlled trials [7, 8, 11].

Morbidity at 6 months

Six months post-surgery was the most concordant time point available across the different trials and for this reason was selected for the analysis of long-term procedure-related morbidity.
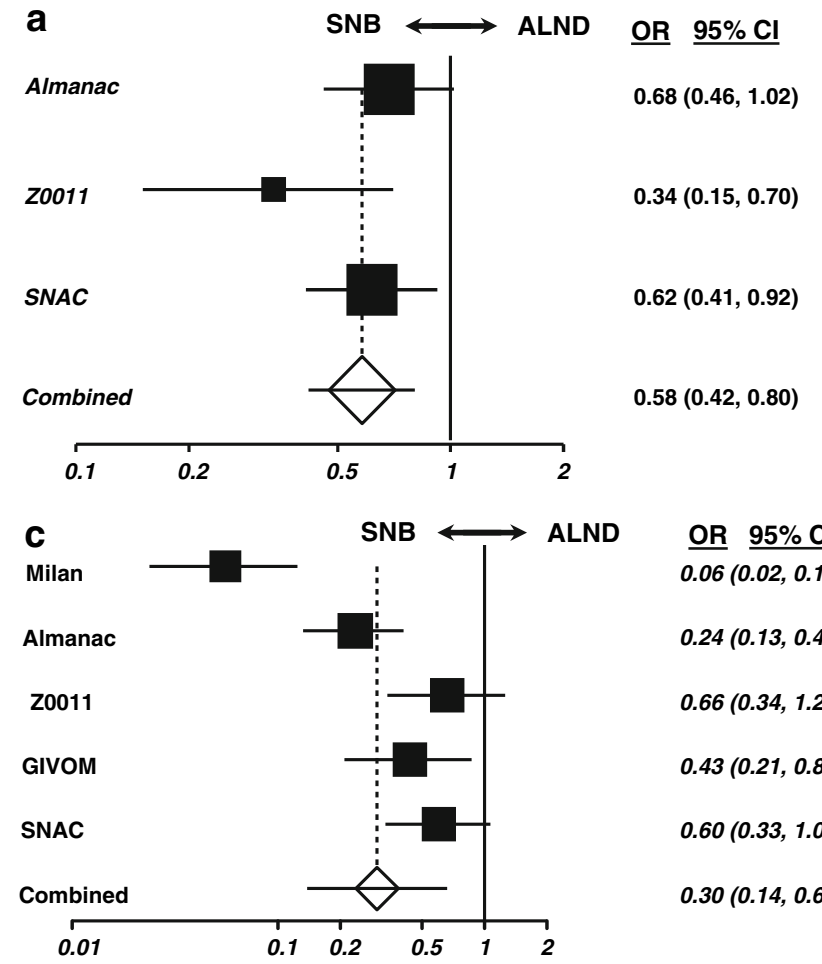

$0.34(0.15,0.70)$

$0.62(0.41,0.92)$

$0.58(0.42,0.80)$

OR $95 \% \mathrm{Cl}$

$0.06(0.02,0.12)$

$0.24(0.13,0.40)$

$0.66(0.34,1.26)$

$0.43(0.21,0.87)$

$0.60(0.33,1.07)$

$0.30(0.14,0.66)$

Fig. 3 Meta-analysis for individual complications following SNB or ALND. Meta-analysis for the outcome of individual complications following axillary staging. In each panel, SNB (sentinel lymph node dissection), left of unity versus ALND (axillary lymph node dissection) and each study is shown by the point estimate of the odds ratio (square proportional to the weight of each study) and $95 \%$ confidence interval $(\mathrm{CI})$ for the odds ratio (extending lines); the combined odds ratio and $95 \%$ confidence intervals by random effects calculations are shown by diamonds. a Wound infection $[n=2,781$,
On examination of data on 2,154 patients from five randomized controlled trials $[3,4,8,9,11]$ a highly significant $70 \%$ reduction in the risk of post-operative arm swelling at 6 months in those patients undergoing a SNB versus ALND was observed $(\mathrm{OR}=0.30,95 \% \mathrm{CI}=0.14$ 0.66, $P=0.0028$ ) (Fig. 3c).

Analysis of 3,265 patients from five randomized controlled trials [3, 4, 7-9] revealed a significant reduction in the risk of post-operative upper limb numbness in those patients undergoing a $\mathrm{SNB}$ versus $\mathrm{ALND}(\mathrm{OR}=0.25$, 95\% CI $=0.10-0.59, P=0.0018$ ) (Fig. 3d).

\section{Potential bias}

The populations were evenly distributed by age, method of cancer detection and hormone receptor status. In four trials the control arm under went SNB followed by routine ALND [3, 8-10]. However, it is possible that inclusion of just seven randomized controlled trials as opposed to all

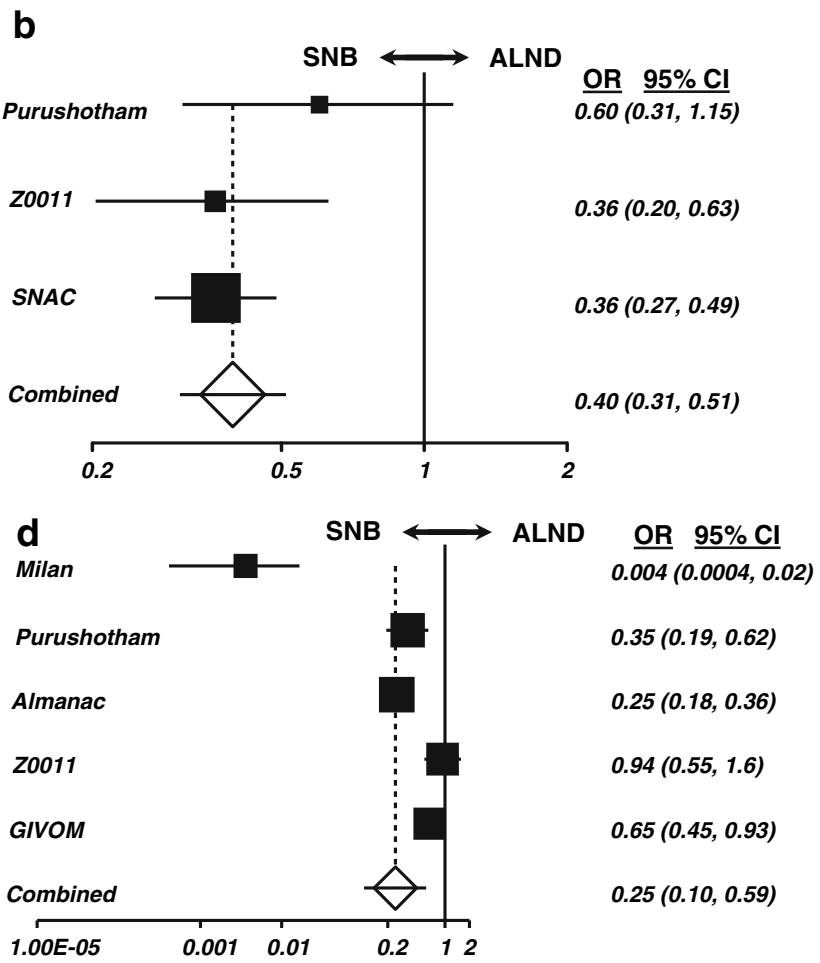

$P=0.0011$, (Test for heterogeneity $Q=3.4(\mathrm{df}=2), P=0.183$, $I^{2}=41.1 \%$ ) $]$, b seroma $[n=2,125, P=0.0071$ (Test for heterogeneity $\left.\left.Q=2.06(\mathrm{df}=2), P=0.357, I^{2}=3 \%\right)\right]$, c limb swelling $[n=2,154, P=0.0028$, (Test for heterogeneity $Q=18.7(\mathrm{df}=4)$, $\left.P=0.0009, I^{2}=78.6 \%\right]$ and d numbness $(n=3,265, P=0.0018$ (Test for heterogeneity $\left.Q=70.0(\mathrm{df}=4), P<0.0001, I^{2}=94.3 \%\right]$. Arrow represents $95 \%$ confidence interval extends beyond the depicted range 
studies examining SNB and ALND may have affected the results, but meta-analyses composed purely of data from randomized studies have a reduced incidence of bias. Furthermore, the maturing but currently unavailable data on morbidity from the NSABP-32 trial which aims to determine if removal of only sentinel nodes provides survival and regional control equivalent to those of ALND, whilst diminishing the magnitude of surgically-related side effects, will also have an impact on the findings of this meta-analysis [12].

The included trials used a variety of methods to assess post-operative limb swelling or lymphoedema. Comparing the circumference of the operated arm with that of the opposite arm was used in three studies [3, 7, 8], patient perception was assessed in three studies [7, 9, 11] the formula for the volume of a truncated cone was used in three studies $[6,9,11]$ and the water displacement method was used in one trial [11]. The gold standard assessment of lymphoedema is using a water displacement technique, but using the formula for the volume of a truncated cone has been shown to be comparable [13]. This analysis may not accurately reflect differences in procedure-related lymphoedema between the two procedures. Lymphedema was assessed at 6 months in the current study as this time point provided the largest number of observations. Petrek et al. have demonstrated that breast cancer-related lymphoedema following ALND occurs maximally in the first 3 years following surgery, however, up to $23 \%$ of patients may still develop arm swelling after this time point [14]. The time course of lymphedema after SNB is not well documented. Considering this, it is possible that longer follow up of patients included in our analysis could result in a greater difference between groups in the risk of development of lymphoedema [15].

\section{Discussion}

This analysis demonstrates that $\mathrm{SNB}$ is equivalent to ALND for the detection of lymph node metastasis in patients with early stage breast cancer and is associated with up to a $75 \%$ reduction in procedure-related morbidity.

Although it is clear that ALND provides no therapeutic benefit to patients with histologically negative nodes and exposes patients to significant potential morbidity, some have advocated ALND as the optimum surgical procedure which is the "gold standard" for staging the axilla [16]. These concerns have been raised by the finding of false negative rates of 5-10\% for SNB when followed by axillary dissection, and have caused some to question its appropriateness in patients at high risk for axillary nodal disease. This meta-analysis comprises all currently available data from randomized trials comparing SNB and
ALND in clinically node negative breast cancer; these results show no difference in the detection of nodal positivity across all trials with a trend towards an improved detection of metastatic lymph nodes when SNB is used. In this analysis, patients undergoing SNB have a $22 \%$ higher odds ratio of having a positive node, a finding that is likely to be due to the more intensive pathologic examination using multiple sections and immunohistochemistry (IHC) which is employed to evaluate the sentinel node and which makes detection of malignant cells more likely [17]. Whilst it is clear that there is a false negative rate associated with identification of incorrect nodes as the sentinel node or failure to identify all of the sentinel nodes, this source of false negative results decreases with increasing experience of the surgeon [18]. In contrast, the false negative rate seen with axillary dissection is due to the inability of the pathologist to perform serial sections and IHC on the 2030 lymph nodes found in an axillary dissection specimen and is not anticipated to decrease, leading us to believe that the staging advantage for SNB observed in this study is likely to increase in the future.

A significant advantage for SNB in the incidence of post-operative wound infection and seroma at 30 days was also confirmed in this analysis. This finding is expected as SNB is less likely to require a large incision and the extensive tissue dissection and lymphatic disruption associated with ALND. The incidence of chronic, potentially disabling complications such as lymphedema and paraesthesia is of greater concern to both patients and surgeons, and their incidence at 6 months post-operatively was also evaluated. Previous studies have reported that lymphoedema of the upper extremity occurs in approximately 5-8\% of patients post-SNB [19-21] and 10-20\% of patients postALND [22]. In the current analysis, the risk of upper limb lymphoedema and paraesthesia at 6 months was significantly diminished in those undergoing SNB (4.8 vs. $13.3 \%$, $\mathrm{SNB}$ vs. ALND). The decreased incidence of lymphoedema is consistent with the removal of a minimal number of axillary nodes and the lesser disruption of the lymphatic drainage of the upper limb with SNB. The documentation of a $75 \%$ reduction in paraesthesias after SNB supports the generalizability of the finding in the ALMANAC trial that the intercostobrachial nerve is four times more likely to be transected during ALND than SNB alone [19].

When all types of post-operative morbidity in these randomized controlled trials were combined, SNB was found to have significantly less associated overall morbidity compared to ALND. This is in keeping with work by Giuliano et al. who demonstrated an overall complication rate of $3 \%$ after SNB compared with $35 \%$ post-ALND [23]. Although it is possible that a SNB followed by an axillary dissection might have greater morbidity than an axillary dissection alone, the ALMANAC trial showed no significant difference 
in post-operative morbidity between SNB (with delayed ALND) and the group who underwent ALND alone [24]. In addition to documented improvements in morbidity, improved psychological well being has been observed in patients who undergo SNB relative to ALND [9].

This study has some limitations; it is based on available published results and the use of updated individual patient data may further enhance the accuracy and reduce the uncertainty of the estimates [25, 26]. Another potential limitation is that the results of recently launched randomized studies were not available to include in the meta-analysis, and data from some published studies could not be extracted. It is also possible that despite some initial training, surgeons may have been developing skills in SNB during the studies included, so the morbidity may reflect still some part of the "learning curve" in this procedure [18].

Our analysis combined data from randomized clinical trials only, to reduce the risk of bias, however, had data from non-randomized studies also been used; a larger cohort would have been achieved. Furthermore, whilst our study selection criteria were stringent, there could be an element of selection bias imposed by their use. However, given the accumulated evidence to-date, the overall summary estimates for the primary outcomes that we considered are unlikely to change.

Despite these caveats, this examination of a large international data set confirms that SNB is the gold standard for staging the axilla in clinically node negative breast cancer. This meta-analysis demonstrates that SNB is at least as accurate as ALND in identifying metastatic lymph nodes and results in a significantly reduced rate of postoperative complication.

\section{Author contributions and Acknowledgements}

Study concept and design: Malcolm R. Kell.

Acquisition of data: John P. Burke, Mitchel Barry.

Analysis and interpretation of data: John P. Burke, Monica Morrow, Malcolm R. Kell.

Drafting of manuscript: John P. Burke, Mitchel Barry, Malcolm R. Kell.

Critical revision: John P. Burke, Monica Morrow, Malcolm R. Kell.

Supervision: Monica Morrow, Malcolm R. Kell.

All authors have approved the final draft submitted. Financial support was gained through the Surgical Oncology Research Appeal, Mater Foundation, Eccles Street, Dublin Ireland.

\section{References}

1. Burke JP, Power C, Gorey TF et al (2008) A comparative study of risk factors and prognostic features between symptomatic and screen detected breast cancer. Eur J Surg Oncol 34(2):149-153

2. Kell MR, Kerin MJ (2004) Sentinel lymph node biopsy. BMJ 328(7452):1330-1331
3. Veronesi U, Paganelli G, Viale G et al (2003) A randomized comparison of sentinel-node biopsy with routine axillary dissection in breast cancer. N Engl J Med 349(6):546-553

4. Mansel RE, Fallowfield L, Kissin M et al (2006) Randomized multicenter trial of sentinel node biopsy versus standard axillary treatment in operable breast cancer: the ALMANAC trial. J Natl Cancer Inst 98(9):599-609

5. Lau J, Ioannidis JP, Schmid CH (1997) Quantitative synthesis in systematic reviews. Ann Intern Med 127(9):820-826

6. www.clinicaltrials.gov

7. Purushotham AD, Upponi S, Klevesath MB et al (2005) Morbidity after sentinel lymph node biopsy in primary breast cancer: results from a randomized controlled trial. J Clin Oncol 23(19):4312-4321

8. Lucci A, McCall LM, Beitsch PD et al (2007) Surgical complications associated with sentinel lymph node dissection (SLND) plus axillary lymph node dissection compared with SLND alone in the American College of Surgeons Oncology Group trial Z0011. J Clin Oncol 25(24):3657-3663

9. Zavagno G, De Salvo GL, Scalco G et al (2008) A randomized clinical trial on sentinel lymph node biopsy versus axillary lymph node dissection in breast cancer: results of the Sentinella/GIVOM trial. Ann Surg 247(2):207-213

10. Krag DN, Anderson SJ, Julian TB et al (2007) Technical outcomes of sentinel-lymph-node resection and conventional axillary-lymph-node dissection in patients with clinically nodenegative breast cancer: results from the NSABP B-32 randomised phase III trial. Lancet Oncol 8(10):881-888

11. Gill G (2009) Sentinel-lymph-node-based management or routine axillary clearance? One-year outcomes of sentinel node biopsy versus axillary clearance (SNAC): a randomized controlled surgical trial. Ann Surg Oncol 16(2):266-275

12. Krag DN, Julian TB, Harlow SP et al (2004) NSABP-32: phase III, randomized trial comparing axillary resection with sentinal lymph node dissection: a description of the trial. Ann Surg Oncol 11(3 Suppl):208S-210S

13. Tewari N, Gill PG, Bochner MA, Kollias J (2008) Comparison of volume displacement versus circumferential arm measurements for lymphoedema: implications for the SNAC trial. ANZ J Surg 78(10):889-893

14. Petrek JA, Senie RT, Peters M, Rosen PP (2001) Lymphedema in a cohort of breast carcinoma survivors 20 years after diagnosis. Cancer 92(6):1368-1377

15. Meric F, Buchholz TA, Mirza NQ et al (2002) Long-term complications associated with breast-conservation surgery and radiotherapy. Ann Surg Oncol 9(6):543-549

16. Silberman AW, McVay C, Cohen JS et al (2004) Comparative morbidity of axillary lymph node dissection and the sentinel lymph node technique: implications for patients with breast cancer. Ann Surg 240(1):1-6

17. Maaskant AJ, van de Poll-Franse LV, Voogd AC et al (2009) Stage migration due to introduction of the sentinel node procedure: a population-based study. Breast Cancer Res Treat 113(1):173-179

18. Classe JM, Curtet C, Campion L et al (2003) Learning curve for the detection of axillary sentinel lymph node in breast cancer. Eur J Surg Oncol 29(5):426-433

19. Fleissig A, Fallowfield LJ, Langridge CI et al (2006) Post-operative arm morbidity and quality of life. Results of the ALMANAC randomised trial comparing sentinel node biopsy with standard axillary treatment in the management of patients with early breast cancer. Breast Cancer Res Treat 95(3):279-293

20. Wilke LG, McCall LM, Posther KE et al (2006) Surgical complications associated with sentinel lymph node biopsy: results from a prospective international cooperative group trial. Ann Surg Oncol 13(4):491-500 
21. McLaughlin SA, Wright MJ, Morris KT et al (2008) Prevalence of lymphedema in women with breast cancer 5 years after sentinel lymph node biopsy or axillary dissection: objective measurements. J Clin Oncol 26(32):5213-5219

22. Erickson VS, Pearson ML, Ganz PA et al (2001) Arm edema in breast cancer patients. J Natl Cancer Inst 93(2):96-111

23. Giuliano AE, Haigh PI, Brennan MB et al (2000) Prospective observational study of sentinel lymphadenectomy without further axillary dissection in patients with sentinel node-negative breast cancer. J Clin Oncol 18(13):2553-2559
24. Goyal A, Newcombe RG, Chhabra A, Mansel RE (2008) Morbidity in breast cancer patients with sentinel node metastases undergoing delayed axillary lymph node dissection (ALND) compared with immediate ALND. Ann Surg Oncol 15(1):262-267

25. Stewart LA, Tierney JF (2002) To IPD or not to IPD? Advantages and disadvantages of systematic reviews using individual patient data. Eval Health Prof 25(1):76-97

26. Clarke M, Stewart L, Pignon JP, Bijnens L (1998) Individual patient data meta-analysis in cancer. Br J Cancer 77(11):20362044 